Volume 136, Number 1, January 2008, Pages 11-19

S 0002-9939(07)09292-1

Article electronically published on September 25, 2007

\title{
ON DIOPHANTINE APPROXIMATION ALONG ALGEBRAIC CURVES
}

\author{
EDWARD B. BURGER AND ASHOK M. PILLAI
}

(Communicated by Wen-Ching Winnie Li)

\begin{abstract}
Let $F(x, y) \in \mathbb{Z}[x, y]$ be a quadratic form such that the associated algebraic curve $\mathcal{C}: F(x, y)=1$ contains a rational point. Here we show that there exists a domain $\mathcal{D} \subseteq \mathbb{R}$ such that for almost all $\xi \in \mathcal{D}$, there exists an infinite sequence of nonzero integer triples $\left(x_{n}, y_{n}, z_{n}\right)$ satisfying the following two properties: $(i)$ For each $n, x_{n} / y_{n}$ is an excellent rational approximation to $\xi$, in the sense that

$$
\lim _{n \rightarrow \infty}\left|\xi y_{n}-x_{n}\right|=0 ;
$$

and (ii) $\left(x_{n} / z_{n}, y_{n} / z_{n}\right)$ is a rational point on the curve $\mathcal{C}$. In addition, we give explicit values of $\xi$ for which both $(i)$ and $(i i)$ hold, and produce a similar result for a certain class of cubic curves.
\end{abstract}

\section{INTRODUCTION}

Given an irrational number $\xi \in \mathbb{R}$, we recall that its associated set of convergents $\left\{p_{n} / q_{n}\right\}$, that is, the collection of rational numbers generated by truncating the continued fraction expansion for $\xi$, is the set of best rational approximations to $\xi$ in terms of the size of their denominators. In particular it is well known that (see, for example, [2] or [5])

$$
\lim _{n \rightarrow \infty}\left|\xi q_{n}-p_{n}\right|=0 .
$$

In this paper we establish, for almost all real $\xi$, the existence of excellent rational approximations that not only satisfy (1.1), but also are connected to rational points on certain algebraic curves. Our first result in this direction is the following.

Theorem 1. Let $F(x, y)=a x^{2}+b x y+c y^{2} \in \mathbb{Z}[x, y]$ be a quadratic form such that the associated algebraic curve $\mathcal{C}: F(x, y)=1$ contains a rational point. Let $\mathcal{D} \subseteq \mathbb{R}$ be the nonempty set defined by $\mathcal{D}=\{x \in \mathbb{R}: F(x, 1)>0\}$. Then for almost all numbers $\xi \in \mathcal{D}$, there exists an infinite sequence of nonzero integer triples $\left(x_{n}, y_{n}, z_{n}\right)$ such that each rational point $\left(x_{n} / z_{n}, y_{n} / z_{n}\right)$ lies on the curve $\mathcal{C}$ and

$$
\lim _{n \rightarrow \infty}\left|\xi y_{n}-x_{n}\right|=0 \text {. }
$$

We note that by the homogeneity of $F$, the rational point $\left(x_{n} / z_{n}, y_{n} / z_{n}\right)$ lies on the curve $\mathcal{C}$ if and only if $F\left(x_{n}, y_{n}\right)=z_{n}^{2}$. We also remark that if

Received by the editors August 1, 2006.

2000 Mathematics Subject Classification. Primary 11J04, 11J70. 
$\operatorname{Disc}(F)=b^{2}-4 a c \leq 0$, then $\mathcal{D}=\mathbb{R}$; and if $\operatorname{Disc}(F)>0$, then $\mathcal{D}$ is either an interval (perhaps unbounded) or the complement of a bounded interval.

We are able to dispense with the set $\mathcal{D}$ if we consider a "norm form" version of Theorem 1. Specifically, we have the following extension.

Theorem 2. Let $F(x, y)=a x^{2}+b x y+c y^{2} \in \mathbb{Z}[x, y]$ be a quadratic form such that the associated curve $\mathcal{C}:|F(x, y)|=1$ contains a rational point. Then for almost all real numbers $\xi$, there exists an infinite sequence of nonzero integer triples $\left(x_{n}, y_{n}, z_{n}\right)$ such that each rational point $\left(x_{n} / z_{n}, y_{n} / z_{n}\right)$ lies on the curve $\mathcal{C}$ and

$$
\lim _{n \rightarrow \infty}\left|\xi y_{n}-x_{n}\right|=0 \text {. }
$$

If we consider the function $F(x, y)=x^{2}+y^{2}$, then Theorem 2 implies that for almost all $\xi \in \mathbb{R}$, there exist infinitely many Pythagorean triples $\left(x_{n}, y_{n}, z_{n}\right)$ such that

$$
\lim _{n \rightarrow \infty}\left|\xi y_{n}-x_{n}\right|=0 .
$$

This special case is due to Elsner [4].

Our method of proof can be applied to produce explicit $\xi$ for which the conclusions of the previous results hold. As an illustration, we offer the following example.

Proposition 3. For a nonnegative integer $b$, let $F(x, y)=x^{2}+b x y+y^{2}$. Given an integer $t>1$, let

$$
\tau=-\tan (2 / t)\left(1+\frac{b}{2} \cot (1 / t)\right) .
$$

Then there exists an infinite sequence of nonzero integer triples $\left(x_{n}, y_{n}, z_{n}\right)$ such that $F\left(x_{n} / z_{n}, y_{n} / z_{n}\right)=1$ and

$$
\lim _{n \rightarrow \infty}\left|\tau y_{n}-x_{n}\right|=0 .
$$

Moreover, we offer the following two diophantine approximation results, the first of which follows immediately from Proposition 3 with $b=0$.

Corollary 4. For any integer $t>1$, there are infinitely many Pythagorean triples $\left(x_{n}, y_{n}, z_{n}\right)$ satisfying

$$
\lim _{n \rightarrow \infty}\left|\tan (2 / t) y_{n}-x_{n}\right|=0 .
$$

Proposition 5. For any integer $t \geq 1$, there exists an infinite sequence of nonzero pairs of integers $\left(x_{n}, y_{n}\right)$ satisfying

$$
\lim _{n \rightarrow \infty}\left|\cosh (1 / t) y_{n}-x_{n}\right|=0
$$

with both $x_{n}+y_{n}$ and $x_{n}-y_{n}$ perfect squares.

In fact the arguments employed in the proofs of Theorems 1 and 2 can be applied to show that Proposition 5 also holds with $\cosh (1 / t)$ replaced by almost all real numbers $\xi$ satisfying $|\xi|>1$.

We recall that a real number $\mathcal{L}$ is a Liouville number if there exist infinitely many pairs of integers $\left(x_{n}, y_{n}\right)$ such that

$$
\left|\mathcal{L} y_{n}-x_{n}\right|<y_{n}^{-n} .
$$

An application of Liouville's celebrated theorem from 1844 on rational approximation to algebraic numbers shows that all Liouville numbers are transcendental (see, 
for example, [3]). If we now turn our attention to diophantine approximation along cubic curves, we have the following result.

Theorem 6. Given an integer $t$, let $\mathcal{C}$ denote the singular cubic curve defined by

$$
\mathcal{C}: y^{2}=x^{3}-3 t^{2} x-2 t^{3} \text {. }
$$

For any given Liouville number $\eta$, let

$$
\xi=\frac{\eta^{2}-2 t}{\eta^{3}-3 t \eta}
$$

Then there are infinitely many distinct triples of nonzero integers $\left(x_{n}, y_{n}, z_{n}\right)$ such that each rational point $\left(x_{n} / z_{n}, y_{n} / z_{n}\right)$ lies on the curve $\mathcal{C}$, and

$$
\lim _{n \rightarrow \infty}\left|\xi y_{n}-x_{n}\right|=0 \text {. }
$$

\section{The Proofs of TheOrems 1 AND 2}

Given a real number $\alpha$, we write

$$
\alpha=a_{0}+\frac{1}{a_{1}+\frac{1}{a_{2}+\frac{1}{\ddots}}}=\left[a_{0}, a_{1}, a_{2}, \ldots\right]
$$

for its simple continued fraction expansion, in which each partial quotient, $a_{n}$, is an integer and for all $n>0, a_{n}>0$. Our arguments will require the following lemma that may be of some independent interest.

Lemma 7. Let $F(x, y)=a x^{2}+b x y+c y^{2} \in \mathbb{Z}[x, y]$ be a homogeneous quadratic form such that there exists a rational point $\left(\lambda_{1}, \lambda_{2}\right)$ satisfying $F\left(\lambda_{1}, \lambda_{2}\right)=1$. If $\xi$ is a real, irrational number such that at least one of the two auxiliary quantities

$$
\frac{\left(\xi a \lambda_{1}-c \lambda_{2}\right) \pm \sqrt{F(\xi, 1)+\xi a c \lambda_{1} \lambda_{2}}}{a \lambda_{1}+(\xi a+b) \lambda_{2}}
$$

is a real, irrational number having unbounded partial quotients, then there exists an infinite sequence of integer triples $\left(x_{n}, y_{n}, z_{n}\right), x_{n} y_{n} z_{n} \neq 0$, such that $F\left(x_{n}, y_{n}\right)=$ $z_{n}^{2}$ and

$$
\lim _{n \rightarrow \infty}\left|\xi y_{n}-x_{n}\right|=0 .
$$

Proof. We write $\lambda_{1}=r / s$ and $\lambda_{2}=t / u$. Clearly, if we consider a line $L$ containing $\left(\lambda_{1}, \lambda_{2}\right)$ and having nonzero rational slope $q / p$, then it must intersect the algebraic curve $\mathcal{C}: F(x, y)=1$ at a second point. If $F(p, q) \neq 0$, then a straightforward calculation reveals that this second point has coordinates

$$
\begin{aligned}
& x=\frac{(-a r u-b s t) p^{2}-2 c s t p q+c r u q^{2}}{s u F(p, q)}, \text { and } \\
& y=\frac{(-b r u-c s t) q^{2}-2 a r u p q+a s t p^{2}}{s u F(p, q)} .
\end{aligned}
$$

Thus if we define

$$
\begin{aligned}
& X=X(p, q)=(-a r u-b s t) p^{2}-2 c s t p q+c r u q^{2}, \\
& Y=Y(p, q)=(-b r u-c s t) q^{2}-2 a r u p q+a s t p^{2}, \text { and } \\
& Z=Z(p, q)=\operatorname{suF}(p, q)
\end{aligned}
$$


then it follows that $F(X, Y)=Z^{2}$. Moreover, we remark that there are only finitely many integer pairs $(p, q)$ for which $X Y Z=0$, and we now confine our analysis to those triples $(X, Y, Z)$ for which $X Y Z \neq 0$.

Inspired by the ratio

$$
\frac{X}{Y}=\frac{(-a r u-b s t)\left(\frac{p}{q}\right)^{2}-2 c s t\left(\frac{p}{q}\right)+c r u}{a s t\left(\frac{p}{q}\right)^{2}-2 a r u\left(\frac{p}{q}\right)+(-b r u-c s t)}
$$

we are led to consider the auxiliary function $G(w)$ defined by

$$
G(w)=\frac{(-a r u-b s t) w^{2}-2 c s t w+c r u}{a s t w^{2}-2 a r u w-(b r u+c s t)} .
$$

Solving the quadratic equation $G(w)=\xi$ yields the following two solutions:

$$
w=\frac{\left(\xi a \lambda_{1}-c \lambda_{2}\right) \pm \sqrt{F(\xi, 1)+\xi a c \lambda_{1} \lambda_{2}}}{a \lambda_{1}+(\xi a+b) \lambda_{2}} .
$$

By our hypothesis, at least one of these two real solutions has unbounded partial quotients. We let $\eta$ denote one such solution; that is, $G(\eta)=\xi$ and the partial quotients of $\eta$ are unbounded. If we write $\eta=\left[a_{0}, a_{1}, a_{2}, \ldots\right]$ and let $p_{n} / q_{n}=$ $\left[a_{0}, a_{1}, \ldots, a_{n}\right]$ denote the $n$th convergent associated with $\eta$, then the following is a well-known inequality from the theory of continued fractions (see [2] or [5]):

$$
\left|\eta-\frac{p_{n}}{q_{n}}\right|<\frac{1}{a_{n+1} q_{n}^{2}} .
$$

We now define the integers

$$
x_{n}=X\left(p_{n}, q_{n}\right), \quad y_{n}=Y\left(p_{n}, q_{n}\right), \quad z_{n}=Z\left(p_{n}, q_{n}\right) .
$$

Thus for all sufficiently large $n, x_{n} y_{n} z_{n} \neq 0$ and $F\left(x_{n}, y_{n}\right)=z_{n}^{2}$. We remark that in view of identity (2.1) in this context we have that

$$
G\left(\frac{p_{n}}{q_{n}}\right)=\frac{x_{n}}{y_{n}} .
$$

Therefore, we conclude that

$$
\left|\xi-\frac{x_{n}}{y_{n}}\right|=\left|G(\eta)-G\left(\frac{p_{n}}{q_{n}}\right)\right| .
$$

Clearly, there exists some $\varepsilon(F, \xi)>0$ such that on the interval $|\eta-w|<\varepsilon(F, \xi)$, $G(w)$ is a differentiable function. Let $N_{0}$ be an integer such that for all $n \geq N_{0}$, $\left|\eta-\frac{p_{n}}{q_{n}}\right|<\varepsilon(F, \xi)$. If we define the interval

$$
I=\left[\eta-\left|\frac{p_{N_{0}}}{q_{N_{0}}}\right|, \eta+\left|\frac{p_{N_{0}}}{q_{N_{0}}}\right|\right],
$$

then for each $n \geq N_{0}, p_{n} / q_{n} \in I$, and thus by the Mean Value Theorem, there exists a real number $\Theta_{n} \in I$ such that

$$
\left|G(\eta)-G\left(\frac{p_{n}}{q_{n}}\right)\right|=\left|G^{\prime}\left(\Theta_{n}\right)\right|\left|\eta-\frac{p_{n}}{q_{n}}\right| .
$$


Since $\left|G^{\prime}(w)\right|$ is a continuous function on $I$, there exists a real number $K_{1}=K_{1}(F, \xi)$ such that $\left|G^{\prime}\left(\Theta_{n}\right)\right| \leq K_{1}$ for all $n \geq N_{0}$. Thus in view of (2.4), (2.5), and (2.3), we conclude that

$$
\left|\xi y_{n}-x_{n}\right|<\frac{K_{1}}{a_{n+1}} \frac{\left|y_{n}\right|}{q_{n}^{2}} .
$$

The definition of $y_{n}$ implies that

$$
\frac{\left|y_{n}\right|}{q_{n}^{2}}=\left|a s t\left(\frac{p_{n}}{q_{n}}\right)^{2}-2 a r u\left(\frac{p_{n}}{q_{n}}\right)-(b r u+c s t)\right|,
$$

which, letting $n \rightarrow \infty$, yields

$$
\lim _{n \rightarrow \infty} \frac{\left|y_{n}\right|}{q_{n}^{2}}=\left|a s t \eta^{2}-2 a r u \eta-(b r u+c s t)\right| .
$$

Hence there exists a constant $K_{2}=K_{2}(F, \xi)$ and an index $N_{1}$ such that for all $n \geq N_{1},\left|y_{n}\right| / q_{n}^{2} \leq K_{2}$. Thus for all $n \geq N_{1}$, inequality (2.6) implies that

$$
\left|\xi y_{n}-x_{n}\right|<\frac{K_{1} K_{2}}{a_{n+1}} .
$$

Since $\eta$ has unbounded partial quotients, there exists a subsequence of indices $n_{i}$ such that

$$
\lim _{i \rightarrow \infty}\left|\xi y_{n_{i}}-x_{n_{i}}\right|=0
$$

with $F\left(x_{n_{i}}, y_{n_{i}}\right)=z_{n_{i}}^{2}, x_{n_{i}} y_{n_{i}} z_{n_{i}} \neq 0$, which completes the proof.

Proof of Theorem 1. Let $\left(\lambda_{1}, \lambda_{2}\right)$ be a rational point satisfying $F\left(\lambda_{1}, \lambda_{2}\right)=1$. Clearly, at least one of $\lambda_{1}$ and $\lambda_{2}$ must be nonzero. If $\lambda_{2}=0$, then we can use the rational point $\left(\lambda_{1}, \lambda_{2}\right)$ to find another rational point on $\mathcal{C}$ having a nonzero $y$ coordinate. Thus without loss of generality, we may assume that $\lambda_{2} \neq 0$. Hence we have that $F\left(\lambda_{1} / \lambda_{2}, 1\right)=1 / \lambda_{2}^{2}>0$, and therefore we see that the set $\mathcal{D}$ is nonempty.

Let $\xi$ be a nonzero element of $\mathcal{D}$; so, $F(\xi, 1)>0$. We note that the line $y=$ $(1 / \xi) x$ intersects the curve $\mathcal{C}$ if and only if $F(\xi x, x)=\xi^{2}$. The previous identity is equivalent to $x^{2}=\xi^{2} / F(\xi, 1)$. Thus the line $y=(1 / \xi) x$ will intersect $\mathcal{C}$ if and only if $\xi$ is a nonzero element of $\mathcal{D}$. If we write $(\tilde{x}, \tilde{y})$ for one of these points of intersection, then we have that $F(\tilde{x}, \tilde{y})=1$ and $\tilde{x} / \tilde{y}=\xi$. Certainly for almost all $\xi \in \mathcal{D}$, the line containing $\left(\lambda_{1}, \lambda_{2}\right)$ and $(\tilde{x}, \tilde{y})$ will have nonzero slope, say $1 / \eta$. We can apply (2.1) and (2.2) as we did in the proof of Lemma 7 to deduce that $G(\eta)=\tilde{x} / \tilde{y}=\xi$. If we solve this equation for $\eta$, then we discover the two real solutions

$$
\frac{\left(\xi a \lambda_{1}-c \lambda_{2}\right) \pm \sqrt{F(\xi, 1)+\xi a c \lambda_{1} \lambda_{2}}}{a \lambda_{1}+(\xi a+b) \lambda_{2}} .
$$

The famous Gauss-Kuz'min distribution on the partial quotients of almost all real numbers (see [5]) immediately implies that almost all real numbers have unbounded partial quotients. Thus for almost all $\xi \in \mathcal{D}$, one of the two real solutions above will have unbounded partial quotients. The theorem now follows from Lemma 7.

Proof of Theorem 2. The proof of Theorem 2 is essentially identical to that of Theorem 1 once we note that for all but at most two real values of $x,|F(x, 1)|>0$. 


\section{The proofs of Propositions 3 And 5}

Proof of Proposition 3. We first recall the well-known continued fraction expansion for $\tan (1 / t)$ (see $[1])$,

$$
\begin{aligned}
\tan (1 / t) & =[0, t-1,1,3 t-2,1,5 t-2,1,7 t-2, \ldots] \\
& =[0, t-1, \overline{1,(2 k+1) t-2}]_{k=1}^{\infty} .
\end{aligned}
$$

If we consider Lemma 7 with the coefficients $a=c=1$ and the rational point $\left(\lambda_{1}, \lambda_{2}\right)=(0,1)$, then we require that $\left.(-1+\sqrt{F(\xi, 1})\right) /(\xi+b)$ has unbounded partial quotients. If we set

$$
\frac{-1+\sqrt{F(\xi, 1)}}{\xi+b}=\tan (1 / t),
$$

make a change of variables $\Omega=\xi+b$, and solve for $\Omega$, we find that

$$
\begin{aligned}
\Omega & =\frac{2 \tan (1 / t)+b}{1-\tan ^{2}(1 / t)}=\tan (2 / t)+\frac{b}{1-\tan ^{2}(1 / t)} \\
& =\tan (2 / t)+\frac{b}{2} \tan (2 / t) \cot (1 / t)=-\tau .
\end{aligned}
$$

Thus by Lemma 7 we conclude that

$$
\lim _{n \rightarrow \infty}\left|-\tau y_{n}-\left(x_{n}+b y_{n}\right)\right|=0,
$$

and $F\left(x_{n}, y_{n}\right)=z_{n}^{2}$. If we define $w_{n}=-\left(x_{n}+b y_{n}\right)$, then it follows that $F\left(w_{n}, y_{n}\right)=$ $z_{n}^{2}$ and

$$
\lim _{n \rightarrow \infty}\left|\tau y_{n}-w_{n}\right|=0
$$

which completes our proof.

Proof of Proposition 5. If set $F(x, y)=x^{2}-y^{2}$ and $\left(\lambda_{1}, \lambda_{2}\right)=(1,0)$, then the auxiliary quantities given in Lemma 7 become $\xi \pm \sqrt{\xi^{2}-1}$. If we now set $\xi+$ $\sqrt{\xi^{2}-1}=e^{1 / t}$, then we find that $\xi=\cosh (1 / t)$. In view of the well-known identities (see [1])

$$
e=[2,1, \overline{2 k, 1,1}]_{k=1}^{\infty} \text { and } e^{1 / t}=[1, \overline{t(2 k-1), 1,1}]_{k=1}^{\infty},
$$

we see that we can apply Lemma 7 in this context. Moreover, the integers $x_{n}, y_{n}$, and $z_{n}$ defined in the proof of Lemma 7 can be expressed as $x_{n}=-p_{n}^{2}-q_{n}^{2}, y_{n}=$ $-2 p_{n} q_{n}$, and $z_{n}=p_{n}^{2}-q_{n}^{2}$. Replacing $\left(x_{n}, y_{n}\right)$ by $\left(-x_{n},-y_{n}\right)$, yields our desired result.

\section{The PRoOF of TheOREM 6}

It is easy to see that the cubic curve $\mathcal{C}: y^{2}=x^{3}-3 t^{2} x+2 t^{3}$ has a singularity (more precisely, a node) at the rational point $(t, 0)$. Thus any line $L$ containing the point $(t, 0)$ and having nonzero rational slope $p / q$ must intersect the curve $\mathcal{C}$ at another point. A straightforward calculation reveals that this new point is

$$
\left(\frac{p^{2}-2 t q^{2}}{q^{2}}, \frac{p^{3}-3 t p q^{2}}{q^{3}}\right) \text {. }
$$


Thus if we define the homogeneous cubic form $F(x, y, z)=y^{2} z-x^{3}+3 t^{2} x z^{2}-2 t^{3} z^{3}$ and let

$$
\begin{aligned}
& X=X(p, q)=p^{2} q-2 t q^{3}, \\
& Y=Y(p, q)=p^{3}-3 t p q^{2}, \quad \text { and } \\
& Z=Z(p, q)=q^{3},
\end{aligned}
$$

then it follows that $F(X, Y, Z)=0$.

If we write $\eta=\left[a_{0}, a_{1}, a_{2}, \ldots\right]$ and let $p_{n} / q_{n}$ be the $n$th convergent associated with $\eta$, then we again have that for all $n$,

$$
\left|\eta-\frac{p_{n}}{q_{n}}\right|<\frac{1}{a_{n+1} q_{n}^{2}} .
$$

We define the integers

$$
x_{n}=X\left(p_{n}, q_{n}\right), \quad y_{n}=Y\left(p_{n}, q_{n}\right), \quad z_{n}=Z\left(p_{n}, q_{n}\right) .
$$

Thus $F\left(x_{n}, y_{n}, z_{n}\right)=0$ and $z_{n} \neq 0$, and hence for all sufficiently large $n$ we have that $x_{n} y_{n} z_{n} \neq 0$ and the point $\left(x_{n} / z_{n}, y_{n} / z_{n}\right)$ lies on the cubic curve $\mathcal{C}$. If we define the auxiliary function $G(w)$ by

$$
G(w)=\frac{w^{2}-2 t}{w^{3}-3 t w},
$$

then it follows that

$$
\left|\xi-\frac{x_{n}}{y_{n}}\right|=\left|G(\eta)-G\left(\frac{p_{n}}{q_{n}}\right)\right| .
$$

As $G(\eta)=\xi$, there exists an $\varepsilon(F, \xi)>0$ and an index $N_{0}$ such that on the interval $|\eta-w|<\varepsilon(F, \xi), G(w)$ is a differentiable function and for all $n \geq N_{0},\left|\eta-\frac{p_{n}}{q_{n}}\right|<$ $\varepsilon(F, \xi)$. We again define the interval

$$
I=\left[\eta-\left|\frac{p_{N_{0}}}{q_{N_{0}}}\right|, \eta+\left|\frac{p_{N_{0}}}{q_{N_{0}}}\right|\right],
$$

and note that for all $n \geq N_{0}, p_{n} / q_{n} \in I$, and thus by the Mean Value Theorem, there exists a real number $\Theta_{n} \in I$ such that

$$
\left|G(\eta)-G\left(\frac{p_{n}}{q_{n}}\right)\right|=\left|G^{\prime}\left(\Theta_{n}\right)\right|\left|\eta-\frac{p_{n}}{q_{n}}\right| .
$$

Since $\left|G^{\prime}(w)\right|$ is a continuous function on $I$, we have that there exists a constant $K_{1}=K_{1}(F, \xi)$ such that $\left|G^{\prime}(w)\right| \leq K_{1}$ for all $w \in I$. Thus $\left|G^{\prime}\left(\Theta_{n}\right)\right| \leq K_{1}$ for all $n \geq N_{0}$ and thus in view of (4.2), (4.3), and (4.1), we conclude that

$$
\left|\xi y_{n}-x_{n}\right|<\frac{K_{1}}{a_{n+1}} \frac{\left|y_{n}\right|}{q_{n}^{2}} .
$$

In view of the definition of $y_{n}$, we have that

$$
\frac{\left|y_{n}\right|}{q_{n}^{2}}=\left|p_{n}\right|\left|\frac{p_{n}^{2}}{q_{n}^{2}}-3 t\right|
$$

and since

$$
\lim _{n \rightarrow \infty}\left|\frac{p_{n}^{2}}{q_{n}^{2}}-3 t\right|=\left|\eta^{2}-3 t\right|,
$$


we conclude that for all sufficiently large $n$, we can bound $\left|y_{n}\right| / q_{n}^{2}$ from above by $K_{2}\left|p_{n}\right|$, for some constant $K_{2}=K_{2}(F, \xi)$. This observation together with inequality (4.4) implies that

$$
\left|\xi y_{n}-x_{n}\right|<K_{1} K_{2} \frac{\left|p_{n}\right|}{a_{n+1}} .
$$

Since $\eta$ is a Liouville number, we know that there exists an infinite subsequence of convergents, $p_{n_{1}} / q_{n_{1}}, p_{n_{2}} / q_{n_{2}}, \ldots, p_{n_{i}} / q_{n_{i}}, \ldots$, satisfying

$$
\left|\eta-\frac{p_{n_{i}}}{q_{n_{i}}}\right|<\frac{1}{\left(q_{n_{i}}\right)^{n_{i}}} .
$$

A well-known identity from the theory of continued fractions (see [2] or [5]) allows us to rewrite the left-hand side of the previous inequality as

$$
\left|\eta-\frac{p_{n_{i}}}{q_{n_{i}}}\right|=\frac{1}{q_{n_{i}}\left(\eta_{n_{i}+1} q_{n_{i}}+q_{n_{i}-1}\right)},
$$

where $\eta_{m}$ is the $m$ th complete quotient of $\eta$, that is, $\eta_{m}=\left[a_{m}, a_{m+1}, \ldots\right]$. Since $\eta_{n_{i}+1}<2 a_{n_{i}+1}$ and $q_{n_{i}-1}<q_{n_{i}}$, we have that

$$
\eta_{n_{i}+1} q_{n_{i}}+q_{n_{i}-1}<3 a_{n_{i}+1} q_{n_{i}}
$$

and thus (4.6) and (4.7) imply that

$$
q_{n_{i}}^{n_{i}-2}<3 a_{n_{i}+1} .
$$

In view of (4.8) and the triangle inequality we deduce that for all sufficiently large $n_{i}$,

$$
\left|p_{n_{i}}\right|=\left|p_{n_{i}}-\eta q_{n_{i}}+\eta q_{n_{i}}\right| \leq q_{n_{i}}\left|\eta-\frac{p_{n_{i}}}{q_{n_{i}}}\right|+q_{n_{i}}|\eta| \leq q_{n_{i}}(1+|\eta|) .
$$

Applying the previous inequality together with inequalities (4.5) and (4.8), yields

$$
\left|\xi y_{n_{i}}-x_{n_{i}}\right|<\frac{3(1+|\eta|) K_{1} K_{2}}{q_{n_{i}}^{n_{i}-3}},
$$

which implies that

$$
\lim _{i \rightarrow \infty}\left|\xi y_{n_{i}}-x_{n_{i}}\right|=0
$$

which completes our proof.

\section{Concluding Remarks}

In view of Mahler's classification of complex numbers (see [3]), we remark that since $\xi$ and the Liouville number $\eta$ from Theorem 6 are algebraically dependent, it follows that $\xi$ is a $U$-number. As the collection of $U$-numbers is a set of measure zero, we are unable to apply Theorem 6 to produce an analogous result for almost all real numbers.

It remains an open problem to determine whether there exists an interval $I$ in which almost all $\xi \in I$ would satisfy Theorem 6 or to produce an example for which no such interval exists. It would also be interesting to determine if there is a version of Theorem 6 for elliptic curves. 


\section{REFERENCES}

1. C. Brezinski, History of Continued Fractions and Padé Approximants, Springer-Verlag, BerlinHeidelberg-New York, 1991. MR1083352 (92c:01002)

2. E.B. Burger, Exploring the Number Jungle: A Journey into Diophantine Analysis, Student Mathematical Library 8, American Mathematical Society, Providence, 2000. MR1774066 (2001h:11001)

3. E.B. Burger and R. Tubbs, Making Transcendence Transparent: An intuitive approach to classical transcendental number theory, Springer-Verlag, Berlin-Heidelberg-New York, 2004. MR2077395 (2005f:11145)

4. C. Elsner, On rational approximations by Pythagorean numbers, Fibonacci Quart. 41 (2003), 98-104. MR1990517 (2004c:11121)

5. A. Ya. Khintchine, Continued Fractions (third edition), Dover Publications, Inc., New York, 1992. MR0161834 (28:5038)

Department of Mathematics, Williams College, Williamstown, Massachusetts 01267

E-mail address: eburger@williams.edu

Department of Mathematics, Williams College, Williamstown, Massachusetts 01267 